\title{
Sleep and hypertension
}

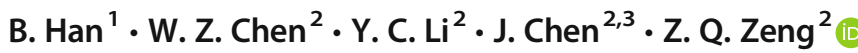 \\ Received: 4 May 2019 /Revised: 28 June 2019 / Accepted: 23 July 2019 / Published online: 12 August 2019 \\ (C) The Author(s) 2019
}

\begin{abstract}
Purpose Hypertension is a global public issue, and sleep status was regarded as its risk factor; however, the results were inconsistent. This study aims to deeply investigate the correlation between sleep status and hypertension.

Methods The electronic databases Cochrane Library, Pubmed, and Embase updated to May 31, 2019, were retrieved. Studies were selected according to the predefined screening criteria, and their qualities were assessed by using quality check scales. Based on Stata 15.1 software, the associations between sleep status and hypertension were analyzed by meta-analyses, using odds ratio and $95 \%$ confidence interval as effect indexes. Furthermore, publication bias and small study bias were evaluated using Begg and Egger's test. In addition, sensitivity analysis was conducted through ignoring one study per time and then observing its influences on the pooled results.

Results A total of 54 studies (involving 1,074,207 subjects) were eligible for this meta-analysis. Six factors were included in this study. Raised blood pressure was associated with obstructive sleep apnea (OSA), oxygen desaturation index (ODI), short sleep duration, and long sleep duration. The differences in $\leq 5 \mathrm{~h}, 6 \mathrm{~h}, \geq 9 \mathrm{~h}$, and $10 \mathrm{~h}$ groups had statistical significances, while there was no significant difference in $\geq 8 \mathrm{~h}$ group. Snoring is a risk factor of hypertension (OR $=1.94,95 \% \mathrm{CI} 1.41-2.67)$. Subgroup analysis was conducted and results were varied.

Conclusions The hypertension risk might be reduced by treated OSA, ODI, and snoring, as well as appropriate sleep duration. More studies with large sample sizes and high qualities should be included to support the findings further.
\end{abstract}

Keywords Sleep $\cdot$ Hypertension $\cdot$ Obstructive sleep apnea $\cdot$ Snoring

\section{Introduction}

Hypertension, also known as high or raised blood pressure, is a condition in which the blood vessels have persistently raised pressure [1]. It is a global public health issue, as well as an important factor for cardiovascular diseases [2-4]. It has been reported that one in four men and one in five women have raised blood pressure. Previous studies reported that hypertension was caused by both genetic and environmental factors.

Electronic supplementary material The online version of this article (https://doi.org/10.1007/s11325-019-01907-2) contains supplementary material, which is available to authorized users.

Z. Q. Zeng

zengyun856@163.com

1 Department of Preventive Medicine, Chengdu Medical College, Chengdu, China

2 Department of Epidemiology and Statistics, Chengdu Medical College, Chengdu, China

3 School of Public Health, Chengdu Medical College, Chengdu, China
Some lifestyle factors, such as smoking, excess salt, drinking, and unhealthy sleep duration, have an effect on the incidence of hypertension [5].

Sleep status alters autonomic nervous system function and other physiologic events that influence blood pressure. In addition, unhealthy sleep status can change the blood pressure response and increase hypertension risk [6]. Habitual sleep duration shorter than the median of 7-8 $\mathrm{h}$ is related to the evaluated incidence of hypertension, which is more common for people sleeping for less than $6 \mathrm{~h}$ per night [7]. Some studies reported that increased sleep time also was a risk factor for hypertension [8]. The association between obstructive sleep apnea (OSA) and hypertension was commonly reported, about $50 \%$ patients with OSA are hypertensive, and an estimated 30 to $40 \%$ of patients with hypertension have OSA [9].

Even though a vast number of studies agreed with that sleep conditions were associated with hypertension, the results of this relationship were not completely consistent in different population. For instance, short sleep duration was found to be a risk factor in American population [8], while the significant association was not reported in Chinese population [10]. In 
terms of sleep apnea, positive association $(\mathrm{OR}=1.72$, $\mathrm{AHI} \geq 30$ ) was found in Malaysian young adults [11], there is, however, no significant results were found in French population [12]. Recent data on the effects of sleep status on hypertension should be explored. There are numerous metaanalyses done on relationship between sleep and hypertension, while the evidence still lacks for comprehensive summary for multiple subtypes of sleep status. Then, to prevent the bias caused by different researches, we performed metaanalyses for sleep status with sufficient available datasets to find cumulative epidemiological evidence for the significant effects contributed dependently or independently by varied subtypes of sleep status to hypertension. This study might lay a theoretical foundation for preventing hypertension.

\section{Methods}

\section{Search strategy and selection criteria}

We systematically searched PubMed, Embase, and Web of Science to identify the association studies (cross-sectional studies, case-control studies, and cohort studies) published before May 31, 2019, using key terms "blood pressure or hypertension or BP or systolic pressure or diastolic pressure or SBP or DBP" and "sleep disorder or sleep duration or sleep apnea or snoring or excessive daytime sleepiness or sleep quality." We also screened references of all included studies, reviews, meta-analyses, pooled analyses, and related citations in PubMed to identify additional publications.

\section{Inclusion and exclusion criteria}

Studies satisfied below criteria were eligible: (1) were published in a peer-reviewed journal in English; (2) reported the associations of sleep status and hypertension; (3) provided risk estimates (e.g., risk ratios (RRs), odd ratios (ORs), 95\% confidence intervals (CIs), regression coefficients and standard errors) or sufficient data to calculate these estimates.

The exclusion criteria were as follows: (1) lacked sufficient information; (2) were not published as full research articles, such as reviews, comments, or letters; (3) were studies on treatment and disease mortality or survival (rather than incidence). Two investigators (Z.ZQ and H.B) assessed the eligibility of each study independently.

\section{Data extraction}

Data extraction was completed by two investigators and subsequently checked by two others. Data collected using the predesigned standard forms, including first author, publishing year, PubMed identifier number, study design, method of case selection, source population, ethnicity of participants, sample size, and risk estimates. When more than one publication used same study population, we collected data from the most recent or the largest one. The references regarding cohort study and case-control study were assessed using Newcastle Ottawa assessment scale, articles focused on cross-sectional study were evaluated using adjusted Newcastle Ottawa assessment scale (see supplementary 4).

\section{Statistical analysis}

We combined the risk estimates of factors from each of the included studies to obtain summary statistics of associations. The RRs or ORs and their 95\% CIs were considered effect indices. Meta-analyses were conducted only for factors with at least three independent datasets. Subgroup analyses were conducted according to ethnicity or study design if the number of datasets was more than five. For studies reporting the categorical results, the risk estimates of factors were calculated using the generalized least squares method by assuming linearity of the natural $\log$-scale ORs. Cochran's $Q$ statistic and $I^{2}$ statistic are used to quantify heterogeneity for the included studies $[13,14]$. For high heterogeneity outcome $\left(I^{2}>50 \%\right)$, the random-effects model was utilized for obtaining the pooled results. For homogeneous outcomes, such as low and moderate heterogeneity $\left(I^{2} \leq 50 \%\right)$, the fixed-effects model was applied to pool the effect indexes. Publication bias of the included studies was evaluated using Begg's test [15]. Potential small study bias was assessed by Egger's test [16]. For sensitivity analysis, one study was ignored at a time and its influences on the pooled results were observed. Statistical analyses were conducted using Stata 15.1 software (https://www.stata.com/).

\section{Results}

\section{Characteristics of eligible studies}

Total 54 publications including 1,074,207 subjects were included in our study (Fig.1). We excluded 3127 irrelevant and duplicate publications. We further excluded 782 articles after title or abstract review, and another 33 articles were not included in our meta-analyses due to non-English languages or insufficient data. Finally, 54 articles (see supplementary 1) including a total of 114 datasets were selected for associations between sleep status and hypertension (see supplementary 2). Among those datasets, 10 datasets including 13,274 subjects were for associations between OSA and hypertension, five datasets including 5,334 subjects were for associations between oxygen desaturation index (ODI) and hypertension, 79 datasets involving 949,489 subjects for association between sleep duration and hypertension, 11 datasets involving 
Fig. 1 Literature search results

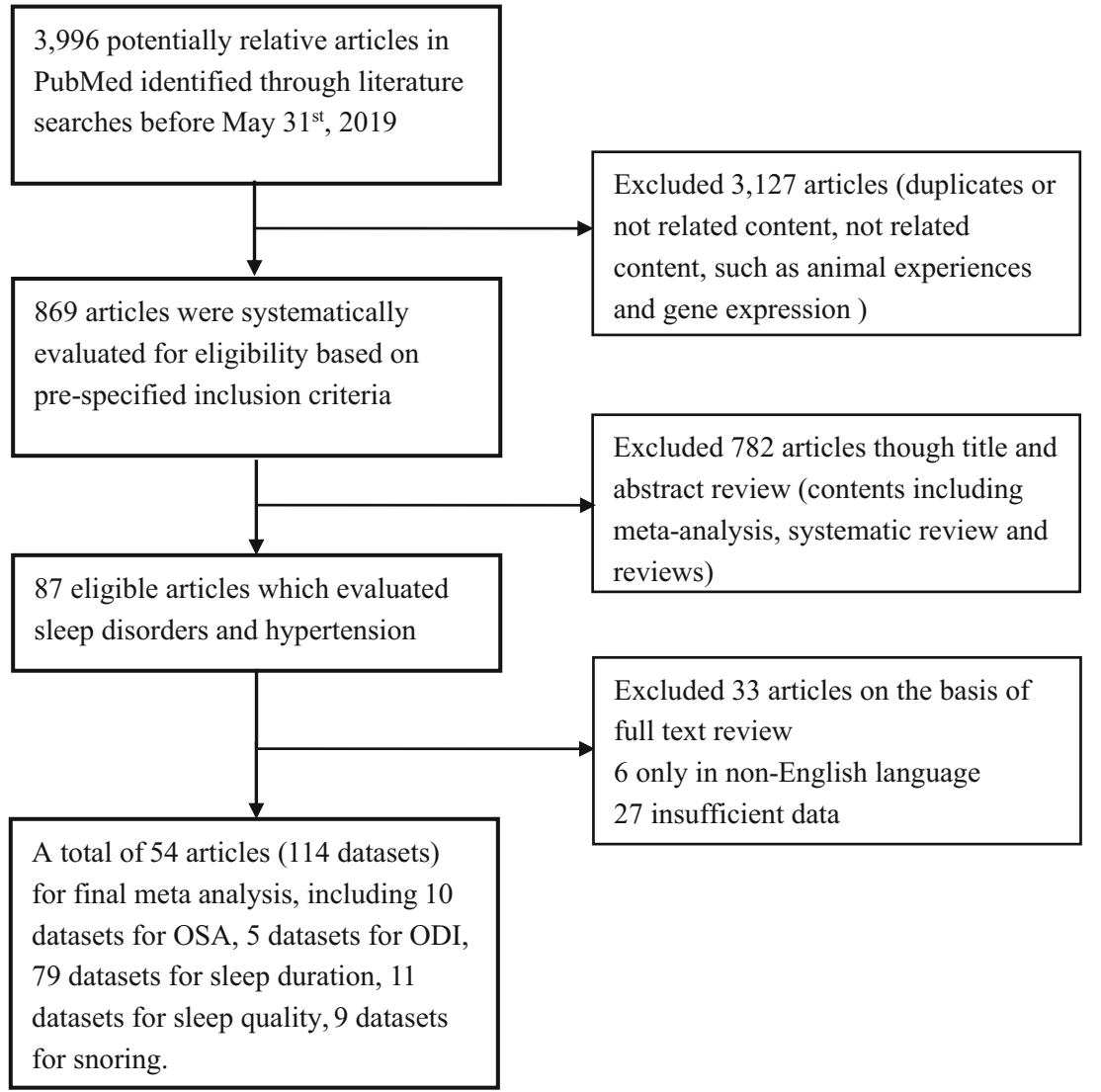

53,897 subjects for association between sleep quality and hypertension, and 5 datasets involving 90,247 subjects for association between snoring and hypertension.

\section{Obstructive sleep apnea and hypertension}

A total of 10 articles were included in the meta-analysis, including two cohort studies, one case-control study, and seven cross-sectional studies. A summary of finding regarding association between OSA and hypertension was shown in Table 1. A significant association between OSA and hypertension was found $(\mathrm{OR}=1.798,95 \% \mathrm{CI} 1.355-2.384)$. Furthermore, we conducted subgroup analysis to evaluate the associations in different study design groups. In cross-sectional study group, the combined results showed OSA was correlated with hypertension significantly $(\mathrm{OR}=1.980,95 \%$ CI 1.312-2.987).

\section{Oxygen desaturation index and hypertension}

As shown in Table 1, five datasets from four articles were considered in analysis. Significant results were found for the association between $3 \%$ oxygen desaturation index and hypertension $(\mathrm{OR}=1.72,95 \% \mathrm{CI} 1.33-2.22)$.

\section{Short sleep duration and hypertension}

Twenty-nine articles were recruited in meta-analysis, including 19 datasets for sleep duration less than or equal to $5 \mathrm{~h}, 18$ datasets for sleep duration less than or equal to $6 \mathrm{~h}$, and 13 datasets for sleep duration less than or equal to $7 \mathrm{~h}$. Sleep duration less than or equal to 5, 6, and $7 \mathrm{~h}$ was associated with hypertension, while in subgroup analysis, for sleep duration less than or equal to $5 \mathrm{~h}$, the significant result was not found in cohort studies (OR $=1.218,95 \%$ CI $0.955-1.491)$. In crosssectional studies, sleep duration less than or equal to 5,6 , and $7 \mathrm{~h}$ was associated with hypertension (Table 1).

\section{Long sleep duration and hypertension}

Fifteen articles were included in meta-analysis, in which, 15 datasets were for sleep duration more than or equal to $8 \mathrm{~h}$, nine datasets for sleep duration more than or equal to $9 \mathrm{~h}$, and five datasets for sleep duration more than or equal to $10 \mathrm{~h}$.

Sleep duration more than or equal $8 \mathrm{~h}$ was associated with hypertension in whole population, while in cohort studies, significant association was not observed $(\mathrm{OR}=1.025$, 95\%CI 0.793-1.326). Sleep duration more than or equal to $9 \mathrm{~h}$ was a risk factor of hypertension $(\mathrm{OR}=1.162,95 \% \mathrm{CI}$ 1.057-1.279), same result was found for sleep duration more 
Table 1 Summarized risk estimate for the association between sleep disorders and hypertension

\begin{tabular}{|c|c|c|c|c|c|c|c|c|}
\hline \multirow[t]{2}{*}{ Factor } & \multirow[t]{2}{*}{ Group } & \multirow[t]{2}{*}{ Datasets } & \multirow[t]{2}{*}{ OR } & \multicolumn{2}{|l|}{$95 \% \mathrm{CI}$} & \multirow[t]{2}{*}{$p$ value } & \multirow[t]{2}{*}{$I^{2}$} & \multirow[t]{2}{*}{$p$ value } \\
\hline & & & & Lower & Upper & & & \\
\hline \multirow[t]{7}{*}{ OSA } & Total & 10 & 1.798 & 1.355 & 2.384 & $4.70 \times 10^{-5}$ & $72.4 \%$ & 0.000 \\
\hline & Asian & 4 & 1.998 & 1.271 & 3.139 & 0.003 & $60.2 \%$ & 0.057 \\
\hline & Caucasian & 5 & 2.054 & 1.093 & 3.862 & 0.025 & $82.4 \%$ & 0.000 \\
\hline & Other & 1 & 1.310 & 1.034 & 1.660 & 0.025 & NA & NA \\
\hline & Case control & 1 & 1.720 & 0.862 & 3.433 & 0.124 & NA & NA \\
\hline & Cohort & 2 & 1.637 & 0.894 & 2.996 & 0.110 & $61.6 \%$ & 0.107 \\
\hline & Cross-sectional & 7 & 1.980 & 1.312 & 2.987 & 0.001 & $79.6 \%$ & 0.000 \\
\hline ODI & Total & 5 & 1.716 & 1.325 & 2.221 & $4.13 \times 10^{-5}$ & $36.8 \%$ & 0.176 \\
\hline \multirow[t]{6}{*}{ Sleep duration $(\leq 5 \mathrm{~h})$} & Total & 19 & 1.448 & 1.252 & 1.674 & $5.73 \times 10^{-7}$ & $90.2 \%$ & 0.000 \\
\hline & African & 1 & 3.480 & 1.692 & 7.158 & 0.001 & NA & NA \\
\hline & Asian & 8 & 1.219 & 1.002 & 1.484 & 0.048 & $64.1 \%$ & 0.050 \\
\hline & Caucasian & 10 & 1.589 & 1.396 & 1.808 & $2.38 \times 10^{-12}$ & $77.1 \%$ & 0.012 \\
\hline & Cross-sectional & 10 & 1.692 & 1.475 & 1.941 & $6.38 \times 10^{-14}$ & $74.5 \%$ & 0.000 \\
\hline & Cohort & 9 & 1.218 & 0.995 & 1.491 & 0.055 & $77.0 \%$ & 0.000 \\
\hline \multirow[t]{6}{*}{ Sleep duration $(\leq 6 \mathrm{~h})$} & Total & 18 & 1.138 & 1.036 & 1.250 & 0.006 & $53.6 \%$ & 0.000 \\
\hline & Asian & 8 & 1.127 & 0.963 & 1.319 & 0.136 & $50.6 \%$ & 0.048 \\
\hline & Caucasian & 9 & 1.181 & 1.050 & 1.329 & 0.006 & $39.3 \%$ & 0.000 \\
\hline & Other & 1 & 0.930 & 0.660 & 1.310 & 0.678 & NA & NA \\
\hline & Cross-sectional & 9 & 1.190 & 1.047 & 1.353 & 0.008 & $61.3 \%$ & 0.008 \\
\hline & Cohort & 9 & 1.077 & 0.941 & 1.231 & 0.282 & $23.2 \%$ & 0.237 \\
\hline \multirow[t]{6}{*}{ Sleep duration $(\leq 7 \mathrm{~h})$} & Total & 13 & 1.196 & 1.064 & 1.344 & 0.003 & $83.4 \%$ & 0.000 \\
\hline & Asian & 9 & 1.081 & 1.016 & 1.150 & 0.014 & $37.0 \%$ & 0.123 \\
\hline & Caucasian & 3 & 1.438 & 0.863 & 2.396 & 0.164 & $90.8 \%$ & 0.000 \\
\hline & Other & 1 & 2.200 & 1.503 & 3.220 & $4.91 \times 10^{-5}$ & NA & NA \\
\hline & Cross-sectional & 10 & 1.207 & 1.051 & 1.387 & 0.008 & $87.4 \%$ & 0.000 \\
\hline & Cohort & 3 & 1.174 & 1.009 & 1.368 & 0.038 & $0.0 \%$ & 0.838 \\
\hline \multirow[t]{5}{*}{ Sleep duration $(\geq 8 \mathrm{~h})$} & Total & 15 & 1.129 & 1.033 & 1.235 & 0.008 & $54.3 \%$ & 0.006 \\
\hline & Asian & 9 & 1.176 & 1.020 & 1.356 & 0.016 & $57.7 \%$ & 0.015 \\
\hline & Caucasian & 6 & 1.086 & 0.931 & 1.266 & 0.258 & $41.2 \%$ & 0.131 \\
\hline & Cross-sectional & 11 & 1.148 & 1.040 & 1.267 & 0.006 & $62.0 \%$ & 0.003 \\
\hline & Cohort & 4 & 1.025 & 0.793 & 1.326 & 0.849 & $30.0 \%$ & 0.232 \\
\hline \multirow[t]{5}{*}{ Sleep duration $(\geq 9 \mathrm{~h})$} & Total & 9 & 1.162 & 1.057 & 1.279 & 0.002 & $39.8 \%$ & 0.102 \\
\hline & Asian & 2 & 1.181 & 1.079 & 1.292 & $3.06 \times 10^{-4}$ & $0.0 \%$ & 0.646 \\
\hline & Caucasian & 7 & 1.094 & 0.884 & 1.354 & 0.410 & $54.1 \%$ & 0.042 \\
\hline & Cross-sectional & 7 & 1.158 & 1.034 & 1.296 & 0.011 & $50.0 \%$ & 0.062 \\
\hline & Cohort & 2 & 1.147 & 0.841 & 1.565 & 0.385 & $21.9 \%$ & 0.258 \\
\hline \multirow[t]{5}{*}{ Sleep duration $(\geq 10 \mathrm{~h})$} & Total & 6 & 1.411 & 1.066 & 1.866 & 0.016 & $90.4 \%$ & 0.000 \\
\hline & Asian & 1 & 1.520 & 1.253 & 1.844 & $2.14 \times 10^{-5}$ & NA & NA \\
\hline & Caucasian & 5 & 1.359 & 0.942 & 1.960 & 0.101 & $92.2 \%$ & 0.000 \\
\hline & Cross-sectional & 1 & 1.410 & 1.332 & 1.492 & $1.91 \times 10^{-32}$ & NA & NA \\
\hline & Cohort & 5 & 1.341 & 0.826 & 2.175 & 0.235 & $92.3 \%$ & 0.000 \\
\hline \multirow[t]{5}{*}{ Sleep quality } & Total & 11 & 1.380 & 1.082 & 1.760 & 0.009 & $96.0 \%$ & 0.000 \\
\hline & Asian & 10 & 1.347 & 1.046 & 1.736 & 0.021 & $96.3 \%$ & 0.000 \\
\hline & Caucasian & 1 & 1.840 & 1.133 & 2.988 & 0.014 & NA & NA \\
\hline & Cross-sectional & 9 & 1.514 & 1.160 & 1.977 & 0.002 & $96.7 \%$ & 0.000 \\
\hline & Case control & 2 & 0.819 & 0.575 & 1.166 & 0.267 & $0.0 \%$ & 0.839 \\
\hline \multirow[t]{5}{*}{ Snoring } & Total & 9 & 1.939 & 1.410 & 2.665 & $4.50 \times 10^{-5}$ & $92.9 \%$ & 0.000 \\
\hline & Asian & 4 & 1.665 & 0.885 & 3.133 & 0.114 & $93.1 \%$ & 0.597 \\
\hline & Caucasian & 5 & 2.186 & 1.578 & 3.029 & $2.48 \times 10^{-6}$ & $78.9 \%$ & 0.520 \\
\hline & Cross-sectional & 6 & 1.917 & 1.106 & 3.323 & 0.020 & $91.4 \%$ & 0.000 \\
\hline & Cohort & 3 & 2.051 & 1.402 & 3.001 & $2.16 \times 10^{-4}$ & $86.8 \%$ & 0.000 \\
\hline
\end{tabular}

the italic numbers means the OR values are statistically significant 
than or equal to $10 \mathrm{~h}$; however, the heterogeneity was very high for this analysis $\left(I^{2}=90.4 \%\right)$.

\section{Snoring and hypertension}

Five articles were eligible for meta-analysis. In total population, snoring was found to be risk factor $(\mathrm{OR}=1.939,95 \% \mathrm{CI}$ 1.410-2.665). Same results were found in cohort studies and in all ethnic subgroups.

\section{Sleep quality and hypertension}

Seven articles reported the association between sleep quality and hypertension. In total population, the significant association was found ( $\mathrm{OR}=1.380,95 \% \mathrm{CI} 1.082-1.760$ ). No significant result was found in case-control studies $(\mathrm{OR}=0.819$, 95\% CI $0.575-1.166$ ).

\section{Heterogeneity, sensitivity analysis, and bias}

As shown in Table 1, the heterogeneity was varied for different factors. Very high heterogeneity was observed in analyses for association of sleep quality, snoring, and sleep duration more than or equal to $10 \mathrm{~h}$. High heterogeneity was found in these meta-analyses. Meta-regression analysis and subgroup analysis were conducted in this study, and high heterogeneity was improved in some subgroups (see supplementary 3 ). We performed sensitivity analysis to evaluate the stability of results and found that the summary ORs did not change, except for the association of sleep duration more than or equal to $10 \mathrm{~h}$ and hypertension. Egger and Begg's tests were conducted to evaluate the publication and small study bias; publication bias and small study bias were found in the association of OSA and hypertension ( $P=0.04$ and 0.01 , respectively).

\section{Discussion}

Hypertension is a major risk factor for cardiovascular diseases. In addition to that, its complications also include heart failure, peripheral vascular disease, renal impairment, retinal hemorrhage, and visual impairment [17]. This meta-analysis was conducted to thoroughly explore the association between sleep status and hypertension. In this study, a total of 54 studies involving 1,074,207 subjects were included, and 114 datasets were analyzed. Six types of sleep status were assessed. The results indicated that OSA, ODI, sleep quality, short or long sleep duration, and snoring were the risk factors of hypertension. However, the association was varied in different subgroups. The publication bias was only found in meta-analysis for association between OSA and hypertension. The sensitivity analysis indicated that the pooled results were stable.
In terms of OSA, positive results were found in all study design groups. For short sleep duration less than or equal to $5 \mathrm{~h}$, it is a risk factor for hypertension in the whole population, which is consistent with previous findings [8]. Notably, in Asian population, the significant result was not observed in analysis; we found there were two studies in China with large sample sizes reported negative results $[10,18]$. Long sleep duration is also considered a factor of hypertension, for sleep duration more than or equal to $8 \mathrm{~h}$, there is no significant result found except in Asian population. However, chronic long sleep duration became a risk factor in whole population when the number of hours is more than $9 \mathrm{~h}$ in adults. Among seven studies regarding snoring, several studies reported that snoring people had a higher risk of hypertension [19-21]. However, some other researchers found that there was no significant association between snoring and hypertension [22]. This study found snoring is an important factor of hypertension with an odds ratio of 1.38 .

The association between sleep status and hypertension may be explained by some potential mechanisms. For OSA, the blood pressure may be attributed to OSAinduced sympathetic activation, which heightens vascular resistance and cardiac output by stimulating the renninangiotensin-aldosterone system [6]. For short sleep duration, some experimental studies have reported that increased blood pressure may be associated with heightened sympathetic nervous system activity after nights. It is also found that short sleep duration can disrupt circadian rhythmicity and autonomic balance [23]. Another hypothesis indicates that depriving sleep hours is a stressful condition, which has been proved to promote salt appetite and suppress renal salt fluid excretion [24], excessive salt intake is confirmed as a risk factor of hypertension. This study searched articles on the relationship between sleep status and hypertension. Then, we conducted the metaanalyses to comprehensively assess the association between them. Even though the results were credible, there were still some limitations. First of all, there are several analyses with high heterogeneity, and it affects the reliability of these results. Secondly, we found the publication bias and small study bias in analysis of association between OSA and hypertension; thus, our results need to be confirmed by more high-quality literatures. Even though these limitations existed, our findings are still valuable because of the large sample size, the comprehensive factors, and the credible analytical methods.

In conclusion, the hypertension risk might be reduced if patients with OSA, ODI could be treated. Chronic short sleep duration or long sleep duration are harmful for hypertension prevention. Moreover, treating snoring is an effective measure to prevent hypertension. However, our findings should be supported by more studies with large sample sizes and high qualities. 
Acknowledgments We gratefully acknowledge all research assistants for their contribution to this study. Han B and Chen WZ contribute equally to this article.

Funding This study was funded by Sichuan Province under grant (number: 16ZA0281) and Collaborative innovation center of elderly care and health under grant (number: YLZBZ1818).

\section{Compliance with ethical standards}

Conflict of interest The authors declare that they have no conflict of interest.

Ethical approval This article does not contain any studies with human participants performed by any of the authors.

Open Access This article is distributed under the terms of the Creative Commons Attribution 4.0 International License (http:// creativecommons.org/licenses/by/4.0/), which permits unrestricted use, distribution, and reproduction in any medium, provided you give appropriate credit to the original author(s) and the source, provide a link to the Creative Commons license, and indicate if changes were made.

\section{References}

1. WHO (2013) A global brief on hypertension: silent killer, global public health crisis (WHO/DCO/WHD/2013.2). World health Orgnization

2. Costa SM, Lima CA, Nobre A, Vieira DMA, Leal ALR (2018) Hypertension bearers with high risk/big risk of cardiovascular diseases and socioeconomic and health indicators. Rev Assoc Med Bras (1992) 64(7):601-610. https://doi.org/10.1590/1806-9282. 64.07.601

3. Li H, Kong F, Xu J, Zhang M, Wang A, Zhang Y (2016) Hypertension subtypes and risk of cardiovascular diseases in a Mongolian population, inner Mongolia, China. Clin Exp Hypertens 38(1):39-44. https://doi.org/10.3109/10641963.2015. 1060981

4. Lu X, Huang J, Wang L, Chen S, Yang X, Li J, Cao J, Chen J, Li Y, Zhao L, Li H, Liu F, Huang C, Shen C, Shen J, Yu L, Xu L, Mu J, Wu X, Ji X, Guo D, Zhou Z, Yang Z, Wang R, Yang J, Yan W, Gu D (2015) Genetic predisposition to higher blood pressure increases risk of incident hypertension and cardiovascular diseases in Chinese. Hypertension 66(4):786-792. https://doi.org/10.1161/ HYPERTENSIONAHA.115.05961

5. Barnett R (2017) Hypertension. Lancet (London, England) 389(10087):2365. https://doi.org/10.1016/S0140-6736(17)315702

6. Calhoun DA, Harding SM (2010) Sleep and hypertension. Chest 138(2):434 443. https://doi.org/10.1378/chest.09-2954

7. Li H, Ren Y, Wu Y, Zhao X (2018) Correlation between sleep duration and hypertension: a dose-response meta-analysis. J Hum Hypertens 33:218-228. https://doi.org/10.1038/s41371-018-01351

8. Grandner M, Mullington JM, Hashmi SD, Redeker NS, Watson NF, Morgenthaler TI (2018) Sleep duration and hypertension: analysis of $>700,000$ adults by age and sex. J Clin Sleep Medicine 14(6): 1031-1039. https://doi.org/10.5664/jcsm.7176
9. Calhoun DA (2010) Obstructive sleep apnea and hypertension. Curr Hypertens Rep 12(3):189-195. https://doi.org/10.1007/ s11906-010-0112-8

10. Song Q, Liu X, Wang X, Wu S (2016) Age- and gender-specific associations between sleep duration and incident hypertension in a Chinese population: the Kailuan study. J Hum Hypertens 30(8): 503-507. https://doi.org/10.1038/jhh.2015.118

11. Asha'ari ZA, Hasmoni MH, Ab Rahman J, Yusof RA, Ahmad RA (2012) The association between sleep apnea and young adults with hypertension. Laryngoscope 122(10):2337-2342. https://doi.org/ 10.1002/lary.23379

12. Faraut B, Touchette E, Gamble H, Royant-Parola S, Safar ME, Varsat B, Leger D (2012) Short sleep duration and increased risk of hypertension: a primary care medicine investigation. J Hypertens 30(7):1354-1363. https://doi.org/10.1097/HJH. $0 \mathrm{~b} 013 \mathrm{e} 32835465 \mathrm{e} 5$

13. Lau J, Ioannidis JP, Schmid CH (1997) Quantitative synthesis in systematic reviews. Ann Intern Med 127(9):820-826

14. Higgins JP, Thompson SG, Deeks JJ, Altman DG (2003) Measuring inconsistency in meta-analyses. BMJ 327(7414):557560. https://doi.org/10.1136/bmj.327.7414.557

15. Begg CB, Mazumdar M (1994) Operating characteristics of a rank correlation test for publication bias. Biometrics 50(4):1088-1101

16. Egger M, Davey Smith G, Schneider M, Minder C (1997) Bias in meta-analysis detected by a simple, graphical test. BMJ 315(7109): 629-634

17. Williams B, Poulter NR, Brown MJ, Davis M, McInnes GT, Potter JF, Sever PS, Thom SM, Bhs guidelines working party ftBHS (2004) British Hypertension Society guidelines for hypertension management 2004 (BHS-IV): summary. BMJ 328(7440):634 640. https://doi.org/10.1136/bmj.328.7440.634

18. Zhang H, Li Y, Mao Z, Liu M, Huo W, Liu R, Liu X, Tu R, Yang K, Qian X, Jiang J, Zhang X, Tian Z, Bie R, Wang C (2018) A doseresponse association of night sleep duration with hypertension in a Chinese rural population: the Henan Rural Cohort Study. J Am Soc Hypertens 12(12):867-879.e863. https://doi.org/10.1016/j.jash. 2018.10.005

19. Lindberg E, Janson C, Gislason T, Svardsudd K, Hetta J, Boman G (1998) Snoring and hypertension: a 10 year follow-up. Eur Respir J 11(4):884-889

20. Kim JS, Song WH, Shin C, Park CG, Seo HS, Shim WJ, Oh DJ, Ryu SH, Rho YM (2001) The prevalence and awareness of hypertension and the relationship between hypertension and snoring in the Korean population. Korean J Intern Med 16(2):62-68

21. Park CG, Shin C (2005) Prevalence and association of snoring, anthropometry and hypertension in Korea. Blood Press 14(4): 210-216. https://doi.org/10.1080/08037050510034248

22. Corbo GM, Forastiere F, Agabiti N, Baldacci S, Farchi S, Pistelli R, Simoni M, Valente S, Viegi G (2006) Rhinitis and snoring as risk factors for hypertension in post-menopausal women. Respir Med 100(8):1368-1373. https://doi.org/10.1016/j.rmed.2005.11.019

23. Kreier F, Yilmaz A, Kalsbeek A, Romijn JA, Sauerwein HP, Fliers E, Buijs RM (2003) Hypothesis: shifting the equilibrium from activity to food leads to autonomic unbalance and the metabolic syndrome. Diabetes 52(11):2652-2656

24. Folkow B (2001) Mental stress and its importance for cardiovascular disorders; physiological aspects, "from-mice-to-man". Scand Cardiovasc J 35(3):163-172

Publisher's note Springer Nature remains neutral with regard to jurisdictional claims in published maps and institutional affiliations. 